\title{
JÁNOS GYÖRGY SZILÁGYI
}

(1918-2016)

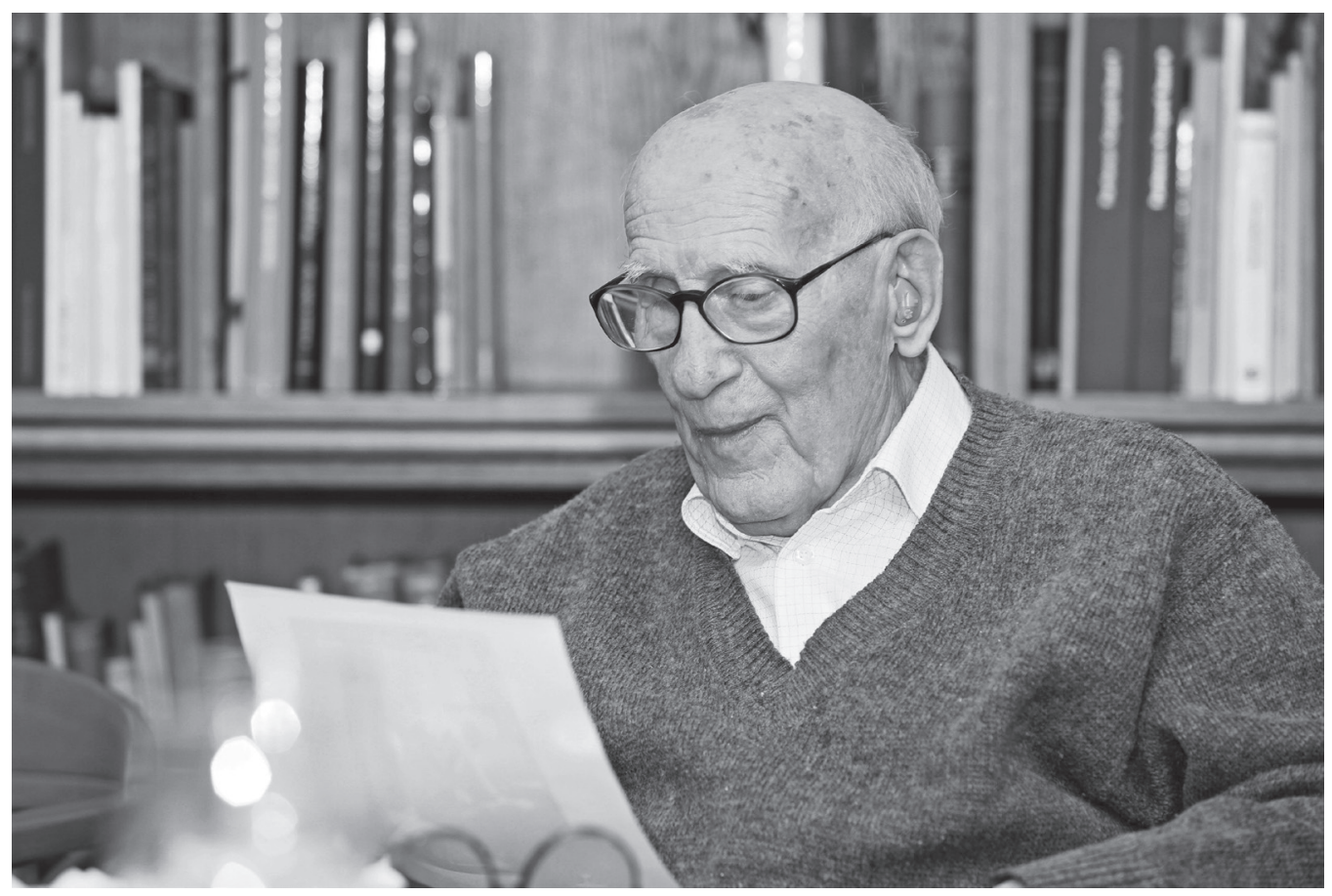

Morto a novantotto anni János György Szilágyi.

La triste notizia, nonostante che ciò paia assolutamente irrazionale, ci ha colti impreparati.

Per cominciare devo chiedermi se esista al mondo qualcuno capace di evocare la sua personalità proteiforme nella sua completa ricchezza? Il famoso storico dell'arte, Ernő Marosi, nel 2000, commentando le sue ricerche e la sua attività intellettuale ha menzionato i seguenti campi in cui egli si è cimentato: studioso dell' antichità e filologo, esperto di museologia, scrittore scientifico e divulgatore dell'oggetto delle sue ricerche, storico della letteratura, traduttore letterario, organizzatore di mostre. Altri lo qualificarono come etruscologo, per absurdum archeologo, ma si guadagnò lodi e apprezzamenti anche come storico delle religioni e semplicemente come docente universitario, anche se il noto poeta ungherese, suo amico, György Somlyó, sostenne che, nel corso della sua lunga e tortuosa carriera la carica di insegnante o professore non era rilevante.

C’è da menzionare però, che nel 1941 egli consegui all’Università di Studi Pázmány Péter di Budapest la laurea di professore di letteratura ungherese e di latino con la tesi intitolata "Studi sull'arte scenica antica" che trattava l'Atellana, la commedia antica scritta in lingua osca, più tardi latina a Roma, che avrebbe potuto segnare anche l'inizio di una gloriosa carriera filologica. La storia e la sorte non lo permisero. Pur essendo ciò un luogo comune, tuttavia è vero, che riuscì a sopravvivere i cinque anni di servizio di lavoro militare e di prigionia di guerra avendo come compagnia Orazio ...

Dopo il ritorno in patria poté reinserirsi nella vita privata aiutato da Aladár Dobrovits, noto egittologo che, nel 1947 offerse a Szilágyi un incarico nella Collezione di Antichità del Museo delle Belle Arti di Budapest. Più tardi, dal 1951 fino al 1992, ricoprì la carica di direttore di tale collezione. Parlando dell'evento forse più decisivo della sua vita disse, "sono divenuto esperto della Collezione di Antichità da autodidatta", per spiegare che lui, il dotto scienziato di filologia divenne col tempo esperto in museografia e uno dei più eminenti studiosi dell'arte antica.

Egli si era proposto un duplice scopo: realizzare il collezionismo basato sul materiale antico già esistente (si tratta principalmente dei marmi antichi e le terrecotte della collezione Arndt) in modo che - e questo era del resto lo scopo principale - la collezione possa rispecchiare ai visitatori del museo nel modo più completo l'arte antica, rivelandone nel frattempo anche l'attualità. Durante i vent'anni di questo periodo veramente emozionante fui l'unico collaboratore di Szilágyi, potendo così osservare da vicino che, pur non cambiando l'angolo visuale, tuttavia la rappresentazione e l'interpretazione di questo ramo dell'arte si approfondirono gradualmente, divenendo sempre più complete. Al fine di suscitare interesse e riflessione volle svelare, anzi, proporre il lato storicamente positivo del falso. Infatti, dal punto di vista dell'interpretazione dell'arte etrusca ciò è tutt'altro che indifferente e ci porta inevitabilmente a percepire il fenomeno della "contraffazione che genera un'opera originale".

Szilágyi si autodefinì principalmente esperto di vasi antichi. Vorrei menzionare in questa sede che, negli anni a cavallo tra il XIX e XX secolo la scoperta che lo studio dei vasi figurati serviva meravigliosamente ad analizzare e studiare i processi della storia della cultura e dell'arte antiche fu un avvenimento decisivo nel campo delle ricerche, portato a successo per opera di Sir John Beazley le cui orme furono seguite dagli 
studiosi e anche l'attività di Szilágyi imboccò questa direzione. Accanto ai vasi a figure nere e rosse di Atene la sua visuale comprendeva, tra l'altro anche la produzione ceramica a figure rosse dell'Italia meridionale, ma principalmente la ceramica etrusca che, rifletteva nel modo più perfetto il suo gusto personale che lo collegava anche al presente, cioè all'arte moderna. Valutando l'arte antica nei suoi scritti espresse spesso la sua preferenza delle forme arcaiche, nonché di quelle anticlassiche, scegliendo come archetipo Exekias l'ateniese, ed eleggendo come campo principale della sua attività scientifica l'arte etrusca, particolarmente la pittura dei vasi figurati. Pubblicò in questo ambito il primo saggio dedicato ai vasi del pittore di Micali, conservati a Budapest (Antiquitas Hungarica III, 1949), per poi segnalare nello studio pubblicato nel 1952 in Acta Antiqua (Zur Frage des etruskischen Handels nach dem Nordens - Contributo alla questione del commercio etrusco diretto verso il Nord) che, desiderava analizzare e interpretare la cultura etrusca nella sua complessità, anzi, non tralasciando nemmeno la sua ambientazione nel contesto europeo.

Tornando però alla questione della ceramica, lo studio dedicato ai vasi, posto in un ambiente nazionale comportava, ovviamente, il compito di pubblicare in una serie tutto il materiale conservato nelle collezioni che appartenevano alla sua sfera d'azione, cioè di pubblicare il Corpus Vasorum Antiquorum (CVA) ungherese. Il fatto che il primo volume venne pubblicato solo nel 1981 naturalmente non è imputabile a Szilágyi, bensì alle difficoltà emerse nell'ambito editoriale, infatti, l'autore aveva portato a termine nel 1970 il manoscritto che doveva servire da base alle pubblicazioni.

Nel primo fascicolo figurava tutto il materiale etrusco conservato nel Museo delle Belle Arti di Budapest, ogni vaso a figure rosse proveniente dalla Campania, inoltre due vasi prodotti in figline sud-italiche non precisamente identificabili e un vaso a figure rosse siciliano. Nota bene, Szilágyi non era solo il più eminente studioso della pittura dei vasi etruschi, ma conosceva a fondo anche la pittura delle figure rosse dei vasi sud-italici. A.D. Trendall ha pubblicato tutta una serie di lavori d'importanza primordiale e se si vuole illustrare la grandiosità delle ricerche condotte da Szilágyi, documentata dalla serie di articoli pubblicati nel Szépmúvészeti Múzeum Közleményei (Bollettino del Museo delle Belle Arti) basti ricordare che egli ha scoperto che, i vasi attribuiti al pittore denominato da Trendall Pittore di Sèvres 50 in realtà fanno parte dell'opera del cosiddetto Pittore della faccia bianca, ciò rivela anche che, lo scopo principale dell'attività scientifica di Szilágyi svolta in questo ambito non era di inserire i vasi facenti parte della collezione antica del Museo delle Belle Arti di Budapest nel sistema creato da Trendall, al contrario, questi vasi in fin dei conti non sono altro per lui che un pretesto per perfezionare l'intero sistema.

Il primo fascicolo ungherese di CVA non è altro che la realizzazione della pubblicazione esemplare di una collezione di vasi minore, e relativamente meno nota, eseguita in base ai principi approvati al Colloquio Internazionale di Lione. La descrizione degli oggetti trattati è più dettagliata del solito e non solo registra tutto ciò che eventualmente non si viene a conoscere dalle semplici fotografie, ma si sofferma anche su particolari che spesso sfuggono anche all'attenzione degli esperti. Nelle pubblicazioni CVA non si trovano descrizioni in cui viene distinta la cosiddetta linea a rilievo da quella elevata, e manca anche la menzione dello schizzo preliminare nonché la presentazione dello stesso in illustrazioni inserite nel testo.

Le questioni iconografiche e la compilazione della bibliografia vengono eseguite secondo le esigenze di un manuale, fatto questo che non è contrario alle richieste formulate nel decreto emanato dal Colloquio Internazionale di Lione in cui si è dichiarato che nel caso delle collezioni nazionali che, costituiscono l'unica possibilità di studio della pittura di vasi antichi il corpus dovrà - incaricandosi anche di funzioni didattiche servire fino a un certo limite anche da manuale della ceramica antica.

In tal modo il primo volume CVA ungherese è caratterizzato da una certa duplicità costruttiva, contenendo sia saggi sui singoli vasi che servono da sostegno per gli studiosi nella loro attività scientifica, e anche studi sui singoli tipi di vasi, corredati da ampi commentari che servono da materiale istruttivo, anzi da manuale sulla ceramica etrusca e sui vasi a figure rosse della Campania per uso e consumo anche del largo pubblico.

Per farla breve, permettetemi di citare la frase conclusiva dell'Introduzione al volume CVA ungherese scritta da P. Devambez, l'ormai purtroppo deceduto direttore internazionale del Corpus Vasorum Antiquorum: "Bien peu de savants du reste consacrent, comme l'a fait M. Szilágyi, des années entières de leur vie à un fascicule dans lequel ils placent toute l'expérience qu'ils ont acquise." (Sono ben pochi, del resto, gli studiosi che, sacrificano anni interi della loro vita a un fascicolo nel quale inseriscono tutta la loro esperienza, acquisita nel corso degli anni.)

Abbiamo menzionato i vasi del Italia meridionale a figure rosse pertanto potremmo dilungarci anche sulle sue "escursioni" fatte in Attica, per recarsi da Lido (Lydos), e dal pittore di Andocide o dal pittore di Brygos, ecc. e sui frutti di questi viaggi che, generalmente destavano un vasto eco nell'ambiente degli studi.

La pubblicazione nel Corpus Vasorum delle collezioni sorte in ambito nazionale pur potendo essere intesa come un compito scolastico, tuttavia l'elaborazione monografica della pittura dei vasi etrusco-corinzi va annoverata a una categoria completamente diversa, invero questa fa parte delle ricerche fondamentali di dimensione internazionale dell'archeologia classica (Ceramica etrusco-corinzia figurata, Firenze).

I due volumi della monografia monumentale che tratta la ceramica etrusco-corinzia furono pubblicati nel 1992 e nel 1998. Quest'opera oltre ad essere una cima delle ricerche e studi di archeologia classica ungherese si è meritata la medesima classifica anche a livello internazionale, elevando e inserendo l'autore nell'illustre schiera composta da nomi come Beazley, Payne, Stibbe e Trendall e altri. Non c'è dubbio, la sintesi serve da prolegomeni per ogni e qualsiasi successiva ricerca del periodo storico della talassocrazia etrusca, avendo chiarito le fondamentali correlazioni sorte entro la storia del Mediterraneo occidentale e aprendo la strada ad una prospettiva attualmente imprevedibile degli studi. Le recensioni e le critiche della sintesi pubblicate nella letteratura professionale rispecchiano per l'appunto questa mia breve valutazione.

Non esiste una questione della scienza dell'archeologia classica alla quale Szilágyi non abbia dato un suo contributo, spesso utilizzando le particolari possibilità offerte dalle recensioni. Grazie alla molteplicità con cui egli praticò la ricerca e gli studi e passando in rivista la bibliografia delle sue opere, talvolta spuntano temi assolutamente insoliti o sorprendenti che oltrepassano di gran lunga i limiti dei temi da lui indagati. Spesso si tratta di analisi dei problemi ormai per così dire classici nell'ambito dell'archeologia classica o degli studi dell'antichità, oppure si ricollegano a scoperte o pubblicazioni recenti. È ben difficile evidenziarne qualsiasi particolare, mentre senza dubbio tutti questi scritti meritano di essere riletti. Da giovane studioso rimasi colpito dalla Szirénzene (Musique de Sirène Bulletin du Musée Hongrois des Beaux-Arts 25, 1964), invece, oggi, volgendo lo sguardo indietro, mi accorgo di preferire in assoluto la pubblicazione intitolata A Pelasg ősök nyomában (in inglese: In search of Pelasgian ancestors, The 1861 Hungarian excavations in the Appennines, 2004). Infatti, quest'opera è veramente frutto di indagini intellettuali, a cui pure io ho potuto partecipare, e nel corso delle quali si venne a sapere che la collezione privata di Budapest era stata scavata 
a Rionero (lato Nord della Lucania) da uno degli antenati della famiglia di cui era originariamente proprietà il materiale scavato e dai suoi soldati. Oltre all'analisi del materiale interessantissimo costituito da vasi a figure rosse, le ricerche ci rivelarono che i soldati ungheresi, lottando capitanati da Garibaldi speravano non solo di contribuire alla liberazione della propria patria dall'oppressore, ma anche di scoprire le proprie origini nel Mediterraneo, nella terra dei Pelasgi.

Molte sue pubblicazioni suscitarono aspre discussioni per poi dare luogo alla opinio communis, forgiata nel purgatorio dei dibattiti. In questa sede desidero menzionare la sua pubblicazione relativa all'iconografia di Iside Pharia che pure suscito grande scalpore e che tuttavia non è altro che una goccia del mare della sua vasta opera (Un problème iconographique - Bulletin de Musée Hongrois des Beaux-Arts 32-33, 1969).

Fin ora non abbiamo nemmeno fatto menzione della pubblicazione sulla situazione attuale e la ricezione dell'arte greca che, ha suscitato largo eco e un vivace dibattito sul tema, e non si è parlato del contributo orientativo concernente l'arte delle province romane includendo in questo contesto anche l'arte pannonica. Il catalogo della mostra presentata nel Museo delle Belle Arti di Budapest, intitolato "La nascita di Roma" (1980), di cui egli scrisse il testo basandosi su pubblicazioni di autori italiani è divenuto ormai un manuale ampiamente impiegato nell' istruzione.

E con ciò siamo giunti a János György Szilágyi nella sua qualità di insegnante a livello universitario che, svolse però questa sua attività principalmente nei locali della Collezione di Antichità del Museo delle Belle Arti che, in questo senso, funzionò come una vera scuola. György Somlyó ha ragione nell'affermare che egli non fu un insegnante o docente universitario nel senso tradizionale della parola, però non si può negare l'alta utilità dell'esercizio professionale svolto entro le mura del Museo delle Belle Arti sotto la sua direzione.

D'altra parte egli fu insegnante nel senso più nobile della parola. Il sistema che adottò e che più gli si confaceva era quello basato sul dialogo, cioè l'insegnamento secondo il metodo socratico, infatti, era noto a tutti che non si rifiutava mai di partecipare a libere conversazioni informali, però, dal punto di vista didattico, preferiva soprattutto il dibattito sui manoscritti freschi, appena portati a termine. Il suo motto diceva: si deve discutere solo i problemi o gli errori, e non vale la pena di parlare di ciò che è evidentemente giusto. Oltre alla precisione professionale esigeva anche l'espressione accurata della questione trattata e amava i pensieri formulati con una certa ricercatezza. Non vado oltre, preferisco citare le parole dell'altro amico poeta: "Szilágyi è l'allenatore che non attenua mai le sue parole biasimanti". Si, infatti, egli fu l'allenatore per eccellenza! E chi sopportava il suo allenamento ne profittava moltissimo, mentre chi non riusciva a sopportarlo, scappava, accettando però le osservazioni, anzi, mi azzardo perfino di dichiarare che riteneva naturale di utilizzare la scienza imparata in un modo nuovo, diverso da tutto ciò che fino allora si aveva conosciuto. Ciò significa che tutti hanno diritto di formare in modo autonomo la propria scienza come lo fece anch'egli imparando il metodo dal Kerényi, da Alföldi o da Moravcsik. Sosteneva che lo studio altro non è che una forma di vivere.

A questo punto forse dovrei fermarmi. Ritengo, però, che non sarebbe giusto non parlare di Szilágyi il filologo. All'inizio della sua carriera la maggior parte dei suoi amici erano giovani scrittori e letterati ed egli cercava di introdurli nella cerchia che circondava il suo maestro, Károly Kerényi, inoltre, l'amico Gábor Devecseri era stato anche suo compagno di corso universitario e questo era l'ambiente, diremmo quasi il campo magnetico in cui nacque l'Omero ungherese (o almeno l'Iliade), o le Metamorfosi di Ovidio, opere (traduzioni) queste, tutte accompagnate dai saggi interpretativi e protettivi di Szilágyi. Non possiamo omettere nemmeno la traduzione dell'Apocolocintosi di Seneca e il relativo saggio, giacché ambedue sono perle preziose dell'opera di Szilágyi. Inoltre, esisteva anche entro la sua attività un punto d'incontro del filologo e dell'archeologo da cui nacquero i due volumi contenenti le fonti scritte dell'arte greca precedente al periodo ellenistico. È un peccato, anzi una perdita irreparabile il fatto che, quest'opera straordinaria sia stata pubblicata solo in lingua ungherese (A görög müvészet világa - Il mondo dell'arte greca, 2 volumi, 1962)

Szilágyi dedicò diversi volumi alla storia degli studi ungheresi dell'antichità, agli eminenti rappresentanti della stessa, agli scopi reali e ipotetici di questi studi. Durante tutta la sua vita fu promotore dell'unità indissolubile degli studi dell'antichità, similmente al suo maestro, Károly Kerényi che esigeva l'incessante e continua osservazione dell'intero mondo antico nella sua complessità, proclamando pure l'indissolubile unità degli studi filologici, storiografici e delle ricerche archeologiche. Non si può rinunciare in nessun modo al compito di portare avanti i nostri studi e ricerche in modo da renderli interessanti, anzi importanti per tutta la società ungherese, indipendentemente dalla situazione attuale degli studi ungheresi dell'antichità.

In base alla sua opera avrebbe potuto ricoprire in Ungheria, o anche all'estero, in qualsiasi università, la carica di titolare della cattedra di filologia greca, o latina, o dell'archeologia antica, ma egli scelse un percorso diverso. Finché il suo stato fisico e la salute glielo permisero egli lavorò giorno e notte nel suo studio situato negli uffici della Collezione di Antichità del Museo delle Belle Arti di Budapest, circondato dai suoi libri prediletti, senza fermarsi nemmeno la domenica o nei giorni festivi.

La sua opera intitolata Paradigmák (Paradigmi) usci nel 1982, ora che non c'è più, che non è più qui con noi, lui stesso è divenuto il paradigma inimitabile dei nostri studi dell’antichità.

Per la bibliografia completa visita is sito www.classics.mfab.hu/antik-gyujtemeny/munkassag-szilagyi

Miklós Szabó 